\title{
A Bimodal Palmprint Verification System
}

\author{
Tai-Kia Tan ${ }^{1}$, Cheng-Leong $\mathrm{Ng}^{1}$, Kar-Ann $\mathrm{Toh}^{2}$, \\ How-Lung Eng ${ }^{2}$, Wei-Yun Yau $^{2}$, and Dipti Srinivasan ${ }^{1}$ \\ ${ }^{1}$ Dept. of Electrical \& Computer Engineering, \\ National University of Singapore, Singapore 117576 \\ dipti@nus.edu.sg \\ ${ }^{2}$ Institute for Infocomm Research, \\ 21 Heng Mui Keng Terrace, Singapore 119613 \\ katoh@ieee.org, \{hleng, wyyau\}@i2r.a-star.edu.sg
}

\begin{abstract}
Hand-based biometrics such as fingerprint and palmprint had been widely accepted because of their convenience and ease in usage without intruding much to one's privacy such as face. The aim of this work is to develop a new point-based algorithm for palmprint feature extraction and perform reliable verification based on the extracted features. This point-based recognition system is next used as part of a bimodal palmprint recognition system combining with a DCT-based (Discrete Cosine Transform) algorithm for identity verification. The performance of the integrated system is evaluated using physical palmprint images.
\end{abstract}

Keywords: Biometrics, Palmprint Recognition, Multimodal Biometrics, and Identity Verification.

\section{Introduction}

Most of the literature reported on palmprint recognition had been based on the use global analysis such as Gabor Filters [1, Discrete Wavelet Transform 2] or global texture energy [3]. Principle lines and wrinkles obtained from edge detectors had also been used directly in some recognition systems [4. Point based approach for palmprint recognition, however, had not been extensively explored except for [5] where paper palmprints were scanned into computer for processing. Main difference between our method and that in [5] is that we use RGB palm images directly captured from a low cost color CCD camera with VGA resolution whereas in 5] a specially designed handprint box was used together with a 200dpi scanner. A simple and yet efficient point-based system is also defined in this paper for palmprint verification. This point-based recognition system will then be combined with a DCT-based method to form a bimodal verification system.

Main contributions are summarized as follows: (i) proposal of a new pointbased method for palmprint verification, (ii) proposal of a bimodal palmprint verification system incorporating the point-based method and a DCT-based method. Some preliminary experiments are reported to show viability of the system. 


\section{System Overview}

A low cost 24-bit colour CCD camera with $768 \times 576$ resolution was used to capture the frontal palm images. The camera was mounted on a customized rig with fluorescence illumination to optimize the image quality. Each user was asked to rest his/her hand on a rigid platform with palm facing a hollow cutout where the camera was positioned within. Apart from an alignment point for the placement of middle finger, no additional alignment pads were used.

\section{Point-Based Verification}

Preprocessing. The preprocessing consists of two main parts - image alignment and image enhancement. Finger gaps are used for the purpose of image alignment in this paper. The palmprint is first binarized using a global threshold. The area of each connected component and the position of its centroid (C1) is determined and used as a criterion for identifying the finger gap objects. After identifying the three finger gap objects, pixels in each finger gap object that are to the right of its corresponding centroid are removed from consideration and a new centroid ( $\mathrm{C} 2)$ of the remaining pixels determined. The process is illustrated in Fig. 11(a). The red dot marks the position of the finger gap object. Pixels that are to the right of the green line are removed from consideration and the centroid of the truncated finger gap object is shown in blue.

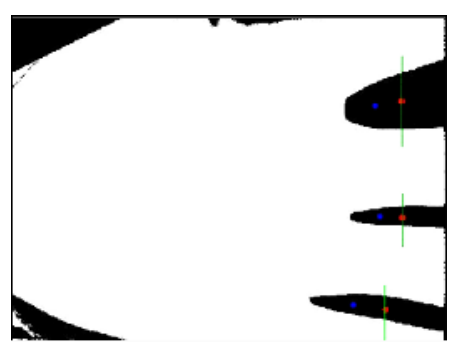

(a)

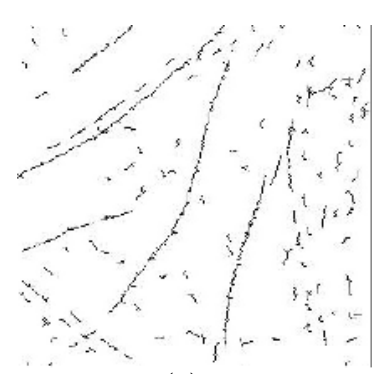

(c)

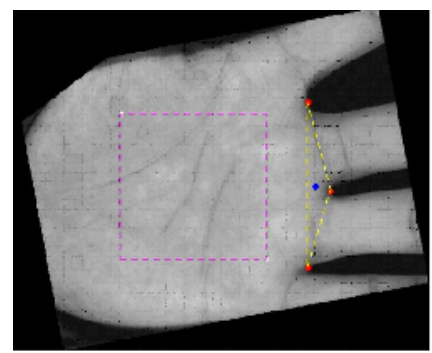

(b)

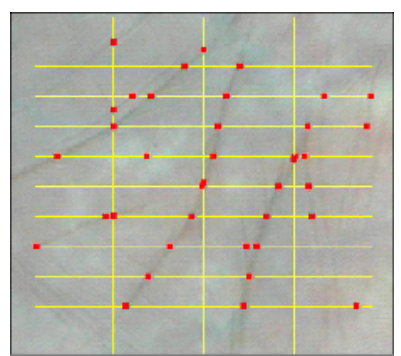

(d)

Fig. 1. (a) Detection of centroids of finger gap objects, (b) Determining ROI from finger webs, (c) ROI after image enhancement, and (d) An example of feature extraction 
Boundary tracing is then carried out. A line that passes through the two centroids (C1 and $\mathrm{C} 2$ ) of each finger gap object is determined and the point of interception between this line and the boundary of the palmprint is recorded as the finger gap location.

The palmprint image is rotated until the finger gap between the index finger and middle finger is directly above the finger gap of the ring finger and last finger. The centroid of the triangle formed by the three finger gap locations is then determined and used as a reference point for defining the Region Of Interest (ROI) of the palmprint. Fig. 1(b) illustrates the above process.

Image enhancement is then carried out on the extracted ROI. A Gaussian filter is first applied to smoothen the image and to remove any inherent noise. At each pixel position, the difference in gray value between the current pixel and its neighbours is calculated. That pixel will be stored as part of the feature line if the difference in gray level is larger then a certain threshold value, $\mathrm{T}$.

As some of the feature lines are poorly contrasted from the skin colour for some palmprint images, an adaptive procedure, which varies the threshold $\mathrm{T}$ dynamically based on the nature of the palmprint image, is adopted. The basis for determining whether the threshold $\mathrm{T}$ needs further adjustment is the number of feature points detected (detection of feature points are presented in next subsection). If too little feature points are detected, it would mean that very few feature lines are detected in the image enhancement procedure. This is a direct consequence of using a threshold that is too high for a particular image. The threshold is therefore lowered and the image enhancement process repeated. The above procedure is iterated until the number of feature points detected is satisfactory.

Line bridging and line thinning is then carried out in order to further improve the quality of the image. Further processing is done to remove lines that do not satisfy the minimum length requirement. Fig. 1(c) shows the ROI after image enhancement.

Feature extraction. Grid lines are superimposed onto the enhanced image. The positions of the points where the gridlines intercept a feature line are recorded as the spatial positions of feature points. The recorded positions are to be used for the computation of error scores. An example of feature extraction is shown in Fig. 11(d).

Orientation information is then computed at each of the detected feature points. The algorithm used for computation of orientation is similar to that proposed by Bazen in [6] for the computation of directional fields for fingerprints. Computation of orientation is done in similar fashion but within a $13 \mathrm{x} 13$ window in the vicinity of feature point instead.

In addition to orientation, the coherence of the feature lines in the vicinity of the feature points can also be computed 6]. If high coherence values are obtained for the feature lines in the vicinity, it would mean that the gradient operator returns largely invariant values for the pixels in the vicinity. This would in turn imply that the feature lines in the region concerned are more or less in the same direction and that there is strong orientation information. Therefore, 
feature points taken at regions with higher coherence values are likely to be more reliable. In this paper, the feature points that have the thirty highest coherence values are used for the computation of error score for each palmprint image.

This orientation information, together with the spatial coordinates of the feature point computed earlier, makes up the feature point vector which characterizes the important information needed for the computation of error score in the next stage.

Point-based matching. After the feature points are detected and its spatial coordinates and orientation information computed and recorded, the error scores are computed. As spatial coordinates and orientation information of the feature points are stored as feature vectors, computation of error scores is based on the Euclidean distances between feature vectors from two different palmprints. The following equations are used to compute the error score between each corresponding pair of feature points.

$$
\begin{gathered}
\theta_{\text {norm }}=k_{1}\left(\alpha / \frac{\pi}{2}\right) \\
\delta_{\text {norm }}=k_{2}\left[\left(x_{1}-x_{2}\right)^{2}+\left(y_{1}-y_{2}\right)^{2}\right]
\end{gathered}
$$

where $\alpha$ is the smaller angle corresponding to the orientation difference of the two feature points. $\left(x_{1}, y_{1}\right)$ and $\left(x_{2}, y_{2}\right)$ are the spatial coordinates of the corresponding pair of feature points. $k_{1}$ and $k_{2}$ are weights attached to orientation and spatial information respectively. The error score contribution by each pair of feature point is given by

$$
\text { Error Score }=\theta_{\text {norm }}+\delta_{\text {norm }}
$$

The total error score for each pair of palmprint images is obtained by summing the error scores of all the thirty pairs of corresponding feature points. A smaller total error score will indicate a better match between the two palmprints.

\section{DCT-Based Verification}

DCT processing. Ahmed, Natarajan, and Rao (1974) first introduced the discrete cosine transform (DCT) in the early seventies. Ever since, the DCT has grown in popularity and several variants have been proposed. In this work, since we have a square image ROI, a 2D DCT is performed on the ROI which is cropped from the grayscale palm-print image. From the resulting coefficients, only a subset of coefficients is chosen such that it can sufficiently represent the palm. A 64 by 64 window of coefficients are obtained from the original 300 by 300 window of coefficients.

The original $64 \times 642 \mathrm{D}$ coefficients map is converted into a $1 \mathrm{D}$ vector by scanning the DCT matrix in a zigzag fashion which is analogous to that of JPEG/MPEG image coding. This is done so that in the $1 \mathrm{D}$ vector, the coefficients are arranged in an order of increasing frequency. 
Feature vector coefficient selection. Even after truncating the coefficients to a smaller window of lower frequency coefficient, the performance of the system is not acceptable. This is because in a 64 by 64 window, there are a total of 4096 coefficients. And not all of them are useful for recognition. Some coefficients are more susceptible to noise, while others are coefficients that characterize a palm image in general and are not distinctive between different palms. For example, the d.c. coefficient is very robust to noise, but it is invariant between different palms, hence it has little use in recognition of palms. In addition, the d.c. coefficient corresponds to the illumination of the image which is not desirable, since illumination plays no part in the recognition of palms. As such, some means of selection of coefficients that can be used for recognition has to be employed.

In order to identify which are the coefficients that are distinct between different palms, we can calculate the variance of that particular coefficient across different palms and select those coefficients with high variance among different palms. On the other hand, to identify which are the coefficients that are robust to noise, we can calculate the variance of that particular coefficient among images from the same palm. From the initial 4096 coefficients of the $64 \times 64$ window, only 2928 coefficients were selected.

Feature matching. To match a particular input palm, the system compares this palm's feature vector to the feature vectors of a palm from the database. The system compares by calculating the Euclidean distance between the two palms. A match is obtained by minimizing this Euclidean distance.

\section{A Bimodal System}

The point-based system was combined with the DCT-based syetms to form a bimodal palmprint verification system. Both parallel and serial integration was attempted, with parallel integration focusing primarily on accuracy and serial integration looking for a compromise between speed and accuracy.

Although the parallel integration method is likely to exhibit greater accuracy, computation is expected to be time consuming as error scores have to be computed for both the point-based algorithm and DCT based algorithm. Serial integration aims to strike a compromise between accuracy and speed by processing the data in two layers. The first layer consists of the DCT-based recognition system. Two predetermined threshold, T1 and T2 are set. After the error score from the DCT-based recognition system have been computed, palmprint images with an error score less than T1 are classified as "genuine users" while those with an error score of more than T2 are classified as "impostors". Only palmprint images that have an error score between $\mathrm{T} 1$ and $\mathrm{T} 2$ are passed to the second layer, which consists of the point-based recognition system, for further classification.

In this paper, $\mathrm{T} 1$ is set at the lowest error score computed for palmprints from impostors using the DCT method while T2 is set at the highest error score computed for palmprints from genuine users using the DCT method. Using these parameters, decisions can be arrived at the first layer for $23.5 \%$ of the palmprints. 


\section{Results}

42,230 error scores had been generated from 206 palmprint images taken from 21 different users using the point-based recognition system. 40,410 are the result of false matches while the remaining 1820 are obtained from genuine matches. These error scores are used to determine the accuracy of the point-based recognition system. An Equal Error Rate of $8.455 \%$ is achieved for the point-based recognition system. The histogram of error score is shown in Fig. 2(a) and the Receiver Operating Characteristics (ROC) of the point-based recognition system is shown in Fig. 2(b). The graph of error rates against error score is shown in Fig. 2(c) below. The point of intersection in the graph constitutes the equal error rate.

109 out of these 206 palmprint images were used to test the accuracy of the bimodal system. Integration of the two recognition systems produced marked improvement in system accuracy. For the set of 109 palmprints used to test the bimodal recognition system, an Equal Error Rate of $9.985 \%$ was achieved for the point-based system while the DCT based system produced an Equal Error Rate of $9.864 \%$. The parallel integrated system is able to achieve an Equal Error Rate of $2.895 \%$ while an Equal Error Rate of $5.965 \%$ is achieved for serial integration. A comparison of the ROC curves is shown in Fig. 2(d). Detailed tabulation of the error rates for each system is given in the Table 1.

(a)

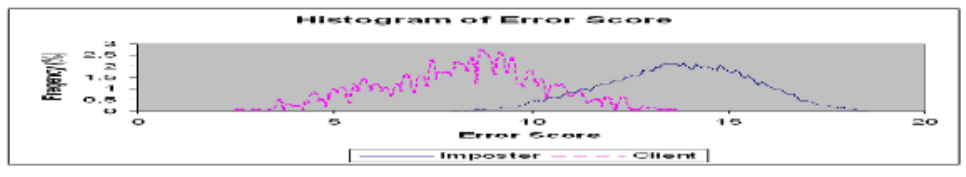

(b)
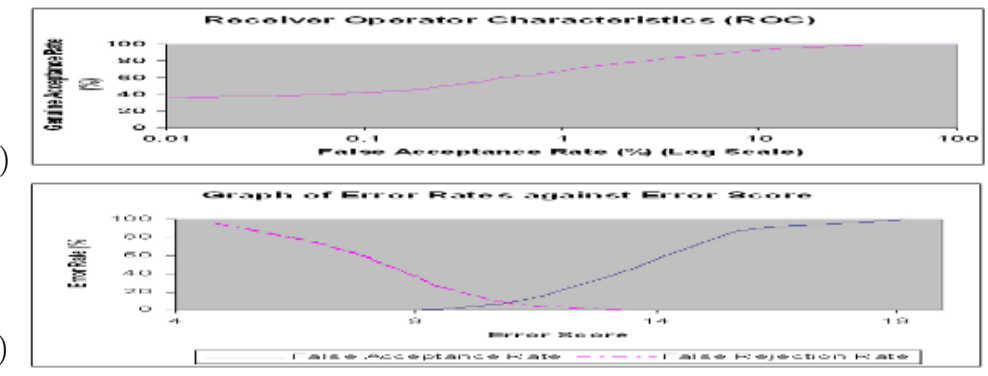

(c)

(d)

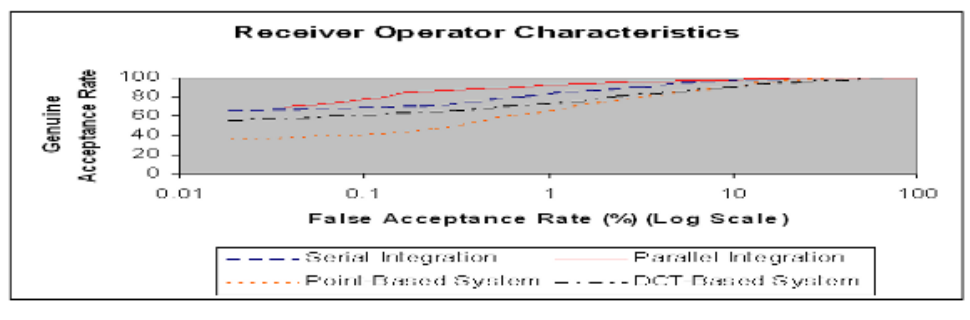

Fig. 2. (a) Histogram of error scores, (b) ROC of point based verification system, (c) Error rates versus error scores, (d) Comparison of ROC curves for different methods 
Table 1. Comparison of Error Rates

\begin{tabular}{|l|c|c|c|c|}
\hline & Point-based & DCT-based & Parallel & Serial \\
\hline EER & $9.99 \%$ & $9.86 \%$ & $2.90 \%$ & $5.97 \%$ \\
FAR $_{F R R=0}$ & $50.48 \%$ & $49.79 \%$ & $29.60 \%$ & $49.80 \%$ \\
FRR $_{F A R=0}$ & $65.64 \%$ & $78.98 \%$ & $34.36 \%$ & $78.87 \%$ \\
\hline
\end{tabular}

\section{Conclusion}

In this paper, a point-based method for palmprint recognition was proposed. The accuracy of the system was observed to give fairly good results on a small database. The system was then extended to form part of a bimodal recognition through serial and parallel integration with a DCT-based method. Accuracy of the bimodal systems were determined and compared with the individual systems. It was found that both serial and parallel integration produced improvements in recognition results with the system utilizing parallel integration faring better then the serially integrated system in terms of accuracy but at the expense of higher computation time. Our immediate future work is to test the system on a large database.

\section{References}

1. D. Zhang, W.-K. Kong, J. You, and M. Wong, "Online palmprint identification," IEEE Trans. Pattern Analysis and Machine Intelligence, vol. 25, no. 9, pp. 10411050, 2003.

2. L. Zhang and D. Zhang, "Characterization of palmprints by wavelet signatures via directional context modeling," IEEE Trans. Systems, Man and Cybernetics, Part-B, vol. 34, no. 3, pp. 1335-1347, June 2004.

3. J. You, W.-K. Kong, D. Zhang, and K. H. Cheung, "On hierarchical palmprint coding with multiple features for personal identification in large databases," IEEE Trans. Circuits and Systems for Video Technology, vol. 14, no. 2, pp. 234-243, 2004.

4. C.-C. Han, H.-L. Cheng, C.-L. Lin, and K.-C. Fan, "Personal authentication using palm-print features," Pattern Recognition, vol. 36, pp. 371-381, 2003.

5. N. Duta, A. K. Jain, and K. V. Mardia, "Matching of palmprints," Pattern Recognition Letters, vol. 23, no. 4, pp. 477-485, 2002.

6. A. M. Bazen and S. H. Gerez, "Systematic methods for the computation of the directional fields and singular points of fingerprints," IEEE Trans. Pattern Analysis and Machine Intelligence, vol. 24, no. 7, pp. 905-919, 2002. 